\title{
Wrangling for microRNAs provokes much crosstalk
}

Ana C Marques*, Jennifer Tan and Chris P Ponting*

\begin{abstract}
Levels of transcripts sharing microRNA response elements are co-regulated. These RNA-RNA interactions imply that combinations of microRNAs modulate cellspecific transcript networks.

Keywords MicroRNAs; competing endogenous RNAs; ceRNAs; PTEN; PTENP1; transcript networks; microRNA sponges; microRNA decoys; competitive endogenous RNAs.
\end{abstract}

Regulated expression of coding and noncoding molecules transcribed from eukaryotic genomes is essential for the differentiation, maintenance and survival of the hundreds of different cell types that constitute a complex multicellular organism. This spatiotemporal regulation of transcript levels requires crosstalk between the genome and its diverse protein and RNA products. Post-transcriptional regulation of gene expression is mediated, in part, by microRNAs (a class of small noncoding RNAs, generally 21 to 25 nucleotides in length), which modulate the transcript levels of thousands of mammalian genes. Mature microRNAs are incorporated into the RNAinduced silencing complex and recognize partially complementary sequence motifs, termed microRNA response elements (MREs), mainly in their target transcripts' 3' untranslated regions ( 3 ' UTRs). This in turn limits target expression levels, usually by repressing protein translation or by mRNA degradation [1].

Four studies [2-5] recently published in Cell show how one transcript can indirectly modulate the cellular abundance of others by affecting the availability of one or more microRNAs (Table 1). Such trans-acting, microRNAdependent properties of transcripts may form the basis of functional networks that involve large numbers of genes.

\section{MicroRNA-mediated crosstalk between transcripts}

A single microRNA has the potential to lower the levels of many transcripts, and target recognition is thought to decrease microRNA concentration. This suggests that

*Correspondence: ana.marques@dpag.ox.ac.uk; chris.ponting@dpag.ox.ac.uk MRC Functional Genomics Unit, University of Oxford, Department of Physiology, Anatomy and Genetics, South Parks Road, Oxford OX1 3PT, UK transcripts sharing MREs co-regulate each other through competitive microRNA binding [6]. The first evidence for crosstalk between endogenous RNAs sharing MREs was found in plants. In Arabidopsis thaliana, a non-protein coding transcript, IPS1 (INDUCED BY PHOSPHATE STARVATION1), and a microRNA, miR-399, are induced following phosphorous starvation. The IPS1 transcript contains a conserved MRE for miR-399, which sequesters the microRNA, thereby releasing PHOS2, a proteincoding gene involved in phosphorous response, from its post-transcriptional regulation by this microRNA [7]. Further examples of noncoding RNAs that act as sponges for endogenous microRNAs have been reported and complement earlier observations that microRNA activity can be efficiently and specifically regulated through the expression of exogenous RNA sponges (reviewed in [8]).

More recently, a retrotransposed gene copy, PTENP1, was shown to compete for microRNAs with its parental gene, PTEN, a known tumor suppressor gene [9]. Transcript levels of this paralogous gene pair are highly correlated in human normal and cancer tissues, suggesting their tight co-regulation [9]. Given that PTENP1 arose from the reverse transcription of PTEN mRNA and subsequent insertion within a different chromosome, its transcription is unlikely to be regulated by duplicated cisregulatory elements. MRE sequences for five of the microRNAs that regulate PTEN are perfectly conserved in PTENP1, suggesting that the observed expression correlation stems from the regulation of the two transcripts by these microRNAs [9]. Two observations are consistent with competition for microRNAs by these two paralogous transcripts: (i) exogenous overexpression of the PTENP1 3' UTR leads to increased PTEN expression in wild-type cells, whereas this is not observed for cells deficient in DICER, an essential component of the microRNA biogenesis pathway [1]; and (ii) decreased expression of PTENP1 has an equivalent effect on PTEN expression and results in similar cellular phenotypes [9]. Analysis of other cancer-related protein-coding/retrocopy pairs revealed that, after duplication, microRNA binding sites are commonly preserved. For example, overexpression of the 3' UTR of KRASP1, a KRAS retrocopy with conserved MREs, leads to increased transcript levels of the parental gene [9]. Despite the apparent lack of a protein-coding open-reading frame, some pseudogenes are transcribed and conserved across diverse species, 
Table 1

\begin{tabular}{lll}
\hline Publication & Findings & Cellular context \\
\hline Tay et al. [2] & $\begin{array}{l}\text { Computational predictions combined with experimental analyses reveal protein-coding } \\
\text { ceRNAs that act as trans-regulators of PTEN with similar tumor-suppressive properties }\end{array}$ & $\begin{array}{l}\text { Human prostate and colon cancer cell } \\
\text { lines }\end{array}$ \\
Karreth et al. [3] & $\begin{array}{l}\text { Transposon insertion within ZEB2 creates a new ceRNA pairing that decreases PTEN } \\
\text { expression levels via crosstalk involving miR-181, miR-200b, miR-25 and miR-92a }\end{array}$ & $\begin{array}{l}\text { Human melanoma cell lines and a mouse } \\
\text { model of melanoma }\end{array}$ \\
Cesana et al. [4] & $\begin{array}{l}\text { Linc-MD1, a muscle-specific long noncoding RNA, regulates muscle differentiation by } \\
\text { acting as a ceRNA that competes with MAML1 and MEF2C, transcripts encoding essential } \\
\text { transcription factors involved in myogenic differentiation, for miR-133 and miR-135 }\end{array}$ & Various mouse and human muscle cell \\
& $\begin{array}{l}\text { Systematic analysis of genome-wide microRNA expression profiles reveals a ceRNA } \\
\text { Sumazin et al. [5] }\end{array}$ & Human glioblastoma cell lines \\
& network that regulates key drivers of gliomagenesis through distinct oncogenic pathways & \\
\hline
\end{tabular}

suggesting that they may have important functional roles. Given that they arose through duplication, it is likely that these retrocopies have preserved ancestral noncoding roles, present also in their parental genes. Therefore, many MRE-containing transcripts will be bifunctional, encoding both a primary, sometimes protein-mediated function and a previously unanticipated post-transcriptional trans-regulatory role.

\section{Networks of competitive endogenous RNAs}

Co-regulated coding or non-coding transcripts that share MREs and crosstalk efficiently have been termed competitive endogenous RNAs (ceRNAs) [8] (Figure 1a). Increases or decreases in the concentration of one targeted transcript (transcript A, Figure 1b) are predicted to result in similar changes in the expression level of the second ceRNA (transcript B, Figure 1b) [8]. Figure 1 illustrates the simplest scenario of crosstalk between two ceRNAs. MicroRNAs target larger numbers of coexpressed transcripts. This generates the potential for more complex networks of trans-acting ceRNAs.

The four recent Cell publications [2-5] provide insights into the mechanisms underlying microRNA-mediated crosstalk among transcripts within ceRNA networks. Development of two computational frameworks for predicting ceRNA transcripts genome-wide [2,5] has now allowed these networks to be investigated in greater detail. In particular, these approaches have identified transcript properties that facilitate efficient crosstalk and ceRNA networks in different tissues. For example, mutually targeted MRE enrichment (MuTaME) [2] evaluates ceRNA candidates by accounting for the number and distribution of shared microRNAs and MREs on the transcripts, and Hermes [5] provides a systematic approach to infer interactions between ceRNA candidates and their targeting microRNAs using genome-wide expression profiles of both the genes and microRNAs. The studies also revealed that in addition to its retrogene PTENP1, expression levels of PTEN were also symmetrically modulated by a set of non-homologous and protein-coding ceRNAs that share with PTEN specific MREs. This ceRNA set included CNOT6L and VAPA in glioblastoma, prostate and cancer cells [2], ZEB2 in melanoma [3], and RB1 in glioblastoma [5]. Together with the muscle-specific long noncoding RNA linc-MD1, which was shown to control muscle differentiation timing in mammalian myoblasts through a similar ceRNAmediated regulation [4], these studies demonstrated that ceRNA networks contain both coding [2,3,5] and noncoding [4] transcripts, and that crosstalk between microRNA co-regulated transcripts is not restricted to paralogous pairs [9], but often involves non-homologous transcripts that share MREs [2-7].

Interactions between ceRNAs seem to be mediated by large numbers of different microRNAs [5], indicating that a change in abundance of a single microRNA is unlikely to have a substantial impact on interactions within these networks [5]. Given that microRNAs often display spatially and temporally restricted expression patterns [1], in each developmental stage and in each cell type, transcript levels are thus likely to be modulated by large numbers of microRNAs. As a consequence the ceRNA network centered on a specific transcript is expected to vary considerably in different cellular contexts. Indeed, in two cancer types (prostate cancer and melanoma) PTEN seems to interact with largely non-overlapping sets of ceRNAs [2,3]. Although variation in expression of either PTEN or PTENP1 led to symmetric changes in the concentration of its paralogous gene [9], this reciprocity is not observed for all ceRNA interactions [2,3]. For example, downregulation of eight ceRNA genes led to a reduction in the expression of PTEN, but comparable changes in PTEN transcript levels affect only half of its predicted ceRNAs [3]. Furthermore, a decrease in PTEN expression levels significantly affected two partially nonoverlapping sets of PTEN ceRNAs in two different melanoma-derived cell lines, WM35 and A375 cells. This indicates that even relatively small changes in cellular environment have a large impact on the components and connectivities of ceRNA networks [3]. 


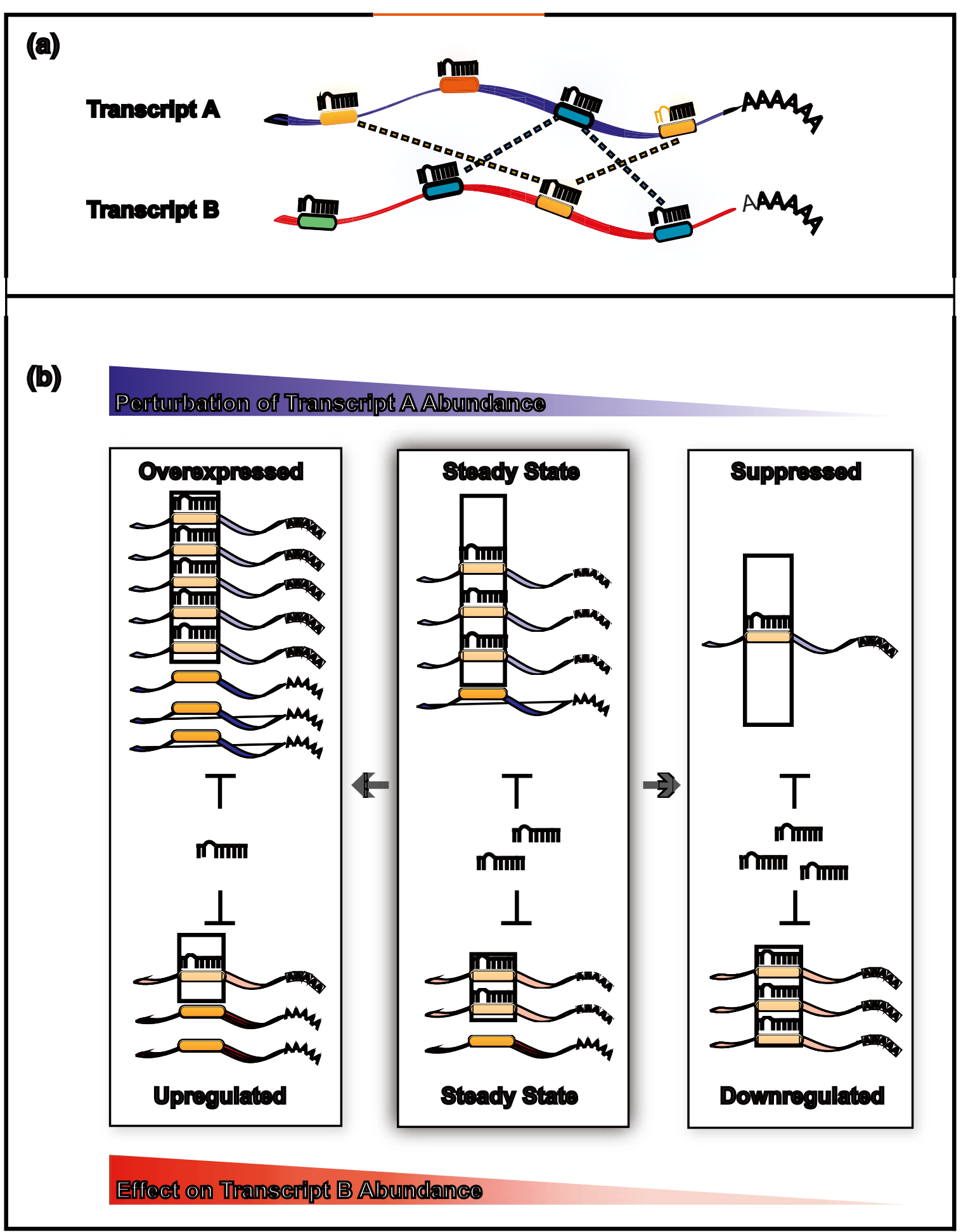

Figure 1. Competitive endogenous RNAs (ceRNAs). (a) Transcripts A (blue) and B (red) are a pair of ceRNAs sharing microRNA response sequences (MREs, boxes) for two microRNAs (blue and yellow). The two ceRNAs can influence the expression level of each other through competitive microRNA binding (dotted lines). MicroRNAs are represented as line structures bound to the MREs. (b) These transcripts co-regulate each other's expression level through competition for shared microRNA (yellow) binding on MREs. In the steady state (middle), ceRNAs and targeting microRNAs are in equilibrium. Overexpression of transcript A (left) reduces the concentration of free microRNAs, thereby increasing expression of transcript B. Decreased expression of transcript A (right) leads to an increase of available microRNAs to bind transcript B and consequently suppresses its expression level. Figure adapted, with permission, from [8]. 


\section{Perspectives}

Four factors seem to influence the efficiency of crosstalk among ceRNAs: (i) relative ceRNA and microRNA concentrations; (ii) numbers of microRNA binding events per ceRNA; (iii) the strength of a microRNA interaction; and (iv) its effect on post-transcriptional regulation [8]. To establish when a ceRNA interaction contributes significantly to the network, and what transcripts are most likely to be affected, it will be important to establish the relative contributions of these factors. Understanding why the effect on expression of interacting transcripts is not reciprocal and how different cellular contexts alter ceRNA networks is likely to shed light on these issues. Several studies, thus far, have focused on PTEN ceRNA networks. This gene is peculiar in two respects: in contrast to other transcripts, its expression is both highly dependent on the levels of its ceRNAs [3] and has readily apparent effects on cell proliferation and on disease association [9]. Future studies on other genes will reveal whether PTEN ceRNA networks are typical or represent important exceptions.

Furthermore, despite the established roles of microRNAs as post-transcriptional regulators, the impact of a microRNA on its target's concentration is usually small, suggesting that many have roles in fine-tuning levels of transcript expression [1]. Similarly, individual ceRNAs are likely to show only limited effects on levels of other transcripts. Clearly, further insights into ceRNA biology will require a greater understanding of the transcripts that constitute these networks and their properties. On one hand, ceRNA networks may allow expression levels of multiple functionally related genes to be concertedly fine-tuned, as indicated by the observed functional enrichments within ceRNA networks [3]. On the other hand, within ceRNA networks, abnormal gene transcription, such as that which occurs in cancer, is probably amplified and thus might become greatly disruptive to normal biological pathways [9]. ceRNAs undoubtedly provide a new layer that adds complexity to gene regulatory networks. Understanding their biological relevance, however, will require further information on how ceRNA networks integrate with other mechanisms of gene expression regulation, and what their relative contributions are to transcript level regulation.

\section{Acknowledgments}

ACM was supported by a Marie Curie IEF fellowship. JT was supported by a Clarendon Award and a NSERC scholarship. CPP was supported by the Medical Research Council and the European Research Council. The authors thank Andrew Bassett and Keith Vance for their insightful comments and suggestions.

\section{Published: 21 November 2011}

\section{References}

1. Bartel DP: MicroRNAs: target recognition and regulatory functions. Cell 2009, 136:215-233.

2. Tay Y, Kats L, Salmena L, Weiss D, Tan SM, Ala U, Karreth F, Poliseno L, Provero P, Di Cunto F, Lieberman J, Rigoutsos I, Pandolfi PP: Coding-independent regulation of the tumor suppressor PTEN by competing endogenous mRNAs. Cell 2011, 147:344-357.

3. Karreth FA, Tay Y, Perna D, Ala U, Tan SM, Rust AG, Denicola G, Webster KA, Weiss D, Perez-Mancera PA, Krauthammer M, Halaban R, Provero P, Adams DJ, Tuveson DA, Pandolfi PP: In-vivo identification of tumor-suppressive PTEN ceRNAs in an oncogenic BRAF-induced mouse model of melanoma. Cell 2011, 147:382-395

4. Cesana M, Cacchiarelli D, Legnini I, Santini T, Sthandier O, Chinappi M, Tramontano A, Bozzoni l: A long noncoding RNA controls muscle differentiation by functioning as a competing endogenous RNA. Cell 2011, 147:358-369.

5. Sumazin P, Yang X, Chiu HS, Chung WJ, Iyer A, Llobet-Navas D, Rajbhandari P, Bansal M, Guarnieri P, Silva J, Califano A: An extensive microRNA-mediated network of RNA-RNA interactions regulates established oncogenic pathways in glioblastoma. Cell 2011, 147:370-381.

6. Seitz H: Redefining microRNA targets. Curr Biol 2009, 19:870-873.

7. Franco-Zorrilla JM, Valli A, Todesco M, Mateos I, Puga MI, Rubio-Somoza I, Leyva A, Weigel D, Garcia JA, Paz-Ares J: Target mimicry provides a new mechanism for regulation of microRNA activity. Nat Genet 2007, 39:1033-1037.

8. Salmena L, Poliseno L, Tay Y, Kats L, Pandolfi PP: A ceRNA hypothesis: the Rosetta Stone of a hidden RNA language? Cell 2011, 146:353-358.

9. Poliseno L, Salmena L, Zhang J, Carver B, Haveman WJ, Pandolfi PP: A codingindependent function of gene and pseudogene mRNAs regulates tumour biology. Nature 2010, 465:1033-1038.

doi:10.1186/gb-2011-12-11-132

Cite this article as: Marques AC, et al.: Wrangling for microRNAs provokes much crosstalk. Genome Biology 2011, 12:132. 